\title{
artigo
}

Xavier, J.M.V.; Olinda, R.A.; Querino, L.A.L.; Barros, M.K.L.V.; Chaves, A.E.P.; Figueiredo, R.A.N.;

Impacto dos fatores meteorológicos na ocorrência de hospitalizações por asma em crianças

\section{Impacto dos fatores meteorológicos na ocorrência de hospitalizações por asma em crianças}

Impact of meteorological factors on the occurrence of hospitalizations for asthma in children

Impacto de los factores meteorológicos en la ocurrencia de hospitalizaciones para el asma en niños

\section{RESUMO}

Objetivos: Caracterizar os casos de asma e identificar os impactos dos fatores meteorológicos na ocorrência de hospitalizações por asma em crianças no município de Campina Grande-PB. Métodos: Para a análise estatística dos dados foi ajustado o modelo binomial negativo, adotando-se um nível de significância de 5\%, com base na plataforma estatística R. Resultados: Pressupõe-se que ocorra um aumento de $81 \%$ nos casos de asma com o aumento da temperatura média, aumentando, do mesmo modo cerca de $27 \%$ desses casos com o aumento da temperatura máxima, em relação à temperatura mínima, espera-se que haja uma diminuição de $26 \%$ dos casos de asma em função do aumento dessa. Estima-se também uma diminuição de $3 \%$ das internações à medida que aumente a umidade relativa do ar. Conclusão: Considerando a influência das variáveis climáticas na saúde na ocorrência das hospitalizações por asma, torna-se possivel planejar ações e implementar medidas preventivas.

DESCRITORES: Variáveis meteorológicas; Doenças respiratórias; Hospitalizações; crianças.

\section{ABSTRACT}

Objectives: To characterize asthma cases and identify the impacts of meteorological factors on the occurrence of hospitalizations for asthma in children in the city of Campina Grande-PB. Methods: For the statistical analysis of the data, the negative binomial model was adjusted, adopting a significance level of $5 \%$, based on the statistical platform R. Results: It is assumed that an increase of $81 \%$ occurs in cases of asthma with an increase in the average temperature, similarly increasing about $27 \%$ of these cases with an increase in the maximum temperature, in relation to the minimum temperature, it is expected that there is a $26 \%$ decrease in asthma cases due to the increase in asthma. A decrease of $3 \%$ in hospitalizations is also estimated as the relative humidity of the air increases. Conclusion: Considering the influence of climatic variables on health in the occurrence of hospitalizations for asthma, it becomes possible to plan actions and implement preventive measures.

DESCRIPTORS: Meteorological variables; Respiratorydiseases; Hospitalizations; kids.

\section{RESUMEN}

Objetivo: Caracterizar casos de asma e identificar los impactos de los factores meteorológicos en la ocurrencia de hospitalizaciones por asma en niños de la ciudad de Campina Grande-PB.Métodos: Para el análisis estadístico de los datos se ajustó el modelo binomial negativo, adoptando un nivel de significancia del 5\%, con base en la plataforma estadística R.Resultados: Se asume que se produce un aumento del $81 \%$ en los casos de asma con un aumento de la temperatura promedio, de igual manera aumentando alrededor del $27 \%$ de estos casos con un aumento de la temperatura máxima, en relación a la temperatura mínima, se espera que hay una disminución del $26 \%$ en los casos de asma debido al aumento de asma.También se estima una disminución del 3\% en las hospitalizaciones a medida que aumenta la humedad relativa del aire.Conclusión: Considerando la influencia de las variables climáticas sobre la salud en la ocurrencia de hospitalizaciones por asma, se hace posible planificar acciones e implementar medidas preventivas.

DESCRIPTORES: Variables meteorológicas; Enfermedades respiratorias; Hospitalizaciones; niños.

RECEBIDO EM: 30/01/2021 APROVADO EM: 02/03/2021

\section{Juliana Meira de Vasconcelos Xavier}

Possui graduação em Enfermagem pela Faculdade Santa Emília de Rodat (2007). Especialista em Saúde da Família (2009) e Saúde Coletiva (2010) pela Faculdades Integradas de Patos-FIP, Especialista em Gestão em Saúde (2012) pela Universidade Estadual da Paraíba-UEPB. Doutora (2019) e Mestre (2015) pelo programa interdisciplinar em Recursos Naturais da Universidade Federal de Campina Grande - UFCG.

ORCID: 0000-0003-0603-1943 


\section{Ricardo Alves de Olinda}

Possui graduação em Estatística pela Universidade Estadual da Paraíba, mestrado em Estatística e Experimentação Agropecuária pela Universidade Federal de Lavras (2008) e doutorado em Estatística e Experimentação Agronômica pela Universidade de São Paulo, Escola Superior de Agricultura "Luiz de Queiroz" (2012).

ORCID: 0000-0002-0509-8428

\section{Luana Andrade Lima Querino}

Graduação em Biologia pela Universidade Federal da Paraíba-UFPB. Especialização em Desenvolvimento e Meio Ambiente-FURNE (2013). Mestrado em Recursos Naturais (2015) e Doutorado em Recursos Naturais pela Universidade Federal de Campina Grande- UFCG (2020). Atua na área deSaúde e meio ambiente.

ORCID: 0000-0001-5874-6006

\section{Mara Karinne Lopes Veriato Barros}

Possui graduação em Bacharelado em Direito pela Universidade Estadual da Paraíba (2008), graduação em Licenciatura em História pela Universidade Federal de Campina Grande (2002), Especialização em Política e Gestão Pública pela Universidade Federal de Campina Grande (2014) e Especialização em Direito do Trabalho e Processo do Trabalho pela Faculdade Damásio de Jesus (2014) e Mestre em Recursos Naturais pela Universidade Federal de Campina Grande (2018).

ORCID: 0000-0003-1215-9599

\section{Ana Elisa Pereira Chaves}

Possui Graduação em Enfermagem e Obstetrícia/Universidade Regional do Nordeste-URNe; Doutorado em Enfermagem/ Universidade Federal do Rio Grande do Norte-UFRN; Mestrado em Enfermagem de Saúde Pública/Universidade Federal da Paraíba-UFPB; Especialização em Educação Profissional na Área de Saúde: Enfermagem/Universidade Federal da ParaíbaUFPB; Especialização em Saúde da Família/Universidade de Pernambuco-UPE; Especialização em Saúde Pública/Universidade de Ribeirão Preto; Especialização em Enfermagem de Saúde Pública/Universidade Federal da Paraíba-UFPB.

ORCID: 0000-0002-1960-0984

\section{Raquel de Almeida Nóbrega Figueiredo}

Possui Técnico em Análises Clínicas pela ENSC (2008), graduação em Ciências Biológicas pela Universidade de Pernambuco (2012), Pós-graduação em Educação Ambiental FIP (2013), Técnico em Hemoterapia pelo CEFOR / RH (2015). Bacharela em Farmácia pela Universidade Estadual da Paraíba (2016).

ORCID: 0000-0001-7297-0936

\section{INTRODUÇÃO}

A asma é uma doença inflamatória crônica das vias respiratórias que torna os brônquios hiperresponsivos aos diversos fatores desencadeantes das crises. É uma doença respiratória que afeta as vias aéreas ou brônquios (tubos que conduzem o ar para dentro dos pulmões), não os brônquios maiores, mas sim as vias aéreas mais delicadas, esses são os brônquios segmentares e os bronquíolos. Por ser uma doença de caráter genético, não pode ser curada, mas sim controlada com sucesso quando o tratamento for adequado ${ }^{(1,2)}$.

A causa exata da asma ainda não é conhecida, mas acredita-se que é causada por um conjunto de fatores:genéticos(história familiar de alergias respiratórias - asma ou rinite) e ambientais. Diversos fatores podem desencadear as crises de asma, tais como infecções virais, poeira domiciliar,
A asma é uma doença inflamatória crônica das vias respiratórias que torna os brônquios hiperresponsivos aos diversos fatores desencadeantes das crises. mofos, odores fortes, umidade, tabagismo, emoções e variações climáticas ${ }^{(1,3)}$.

As crianças asmáticas possuem maior suscetibilidade aos efeitos dos fatores ambientais, pois há aumento da hiperresponsividade brônquica e da sensibilidade a agentes do meio ambiente.Tal fato pode ser explicado pela imaturidade do sistema imunológico que pode ser ainda mais reprimido no período do inverno, as baixas temperaturas promovem espasmos do trato respiratório e isquemia devido a contração capilar em crianças, resultando em enfraquecimento do movimento ciliar e consequentemente dificuldade na remoção de vírus e bactérias no epitélio respiratório ${ }^{(4,5)}$.

Contudo os efeitos dos fatores climáticos no nascimento diminuem com o passar do tempo, à medida que a criança cresce, sendo compatíveis com o desenvolvimento do sistema imunológico e o aumento de diâmetro das vias respiratórias nos primeiros anos de vida ${ }^{(6)}$. 
Diversos estudos em todo mundo têm correlacionado às condições climáticas com morbidade e mortalidade, por diversas doenças. Algumas variáveis climáticas, tais como: temperatura do ar, umidade relativa e precipitação pluviométrica, demostram interferir na ocorrência de doenças respiratórias ${ }^{(7)}$.

Diante do exposto percebe-se que as oscilações em variáveis meteorológicas concorrem de forma efetiva para ocorrência de doenças respiratórias no ser humano, em especial nas crianças. Objetivou-se asma em crianças menores de nove anos e identificar quais os impactos dos fatores meteorológicos na ocorrência de hospitalizações no município de Campina Grande- PB. O período de estudo compreendeu a janeiro de 1998 a dezembro de 2017.

\section{MÉTODOS}

Área e período do estudo - Esta pesquisa foi realizada com dados relativos ao município de Campina Grande, localizado no Estado da Paraíba (PB), do período de janeiro de 1998 a dezembro de 2017, equivalente há vinte anos. $\mathrm{O}$ município de Campina Grande está a uma altitude média de 555 metros com esta pesquisa caracterizar os casos de

acima do nível do mar, distante a $120 \mathrm{~km}$ da Capital do Estado, João Pessoa. A área do município de Campina Grande abrange 593,026 $\mathrm{Km}^{2}$, contando com uma população estimada em 407.754 habitantes, segundo dados do Instituto Brasileiro de Geografia e Estatística possui um clima com temperaturas mais moderadas, considerado Tropical com estação seca. A estação chuvosa se inicia em maio com término em setembro, podendo se estender até outubro (IBGE, 2018).

A atual pesquisa utilizou um estudo transversal de abordagem quantitativa e descritiva. Os dados foram de fonte secundária, relativos às séries de dados meteorológicos obtidas no Instituto $\mathrm{Na}$ cional de Meteorologia (INMET) e do número de hospitalizações por asma os dados epidemiológicos foram coletados no Departamento de informática do SUS-DATASUS, através do Sistema de Informações Hospitalares (SIH). A pesquisa está de acordo com a Resolução $\mathrm{n}^{\circ}$ 510/16 do Conselho Nacional de Saúde que dispõem sobre pesquisas que utilizam informações de acesso público, otimiza os procedimentos de avaliação, que isentam a necessidade de aprovação prévia pelo sistema CEP/CONEP. Sendo esses, dados administrativos obtidos de documentação

Figura 1 - Distribuição do número de casos de asma em crianças menores de 9 anos no período de 1998 a 2017, Campina Grande (PB).

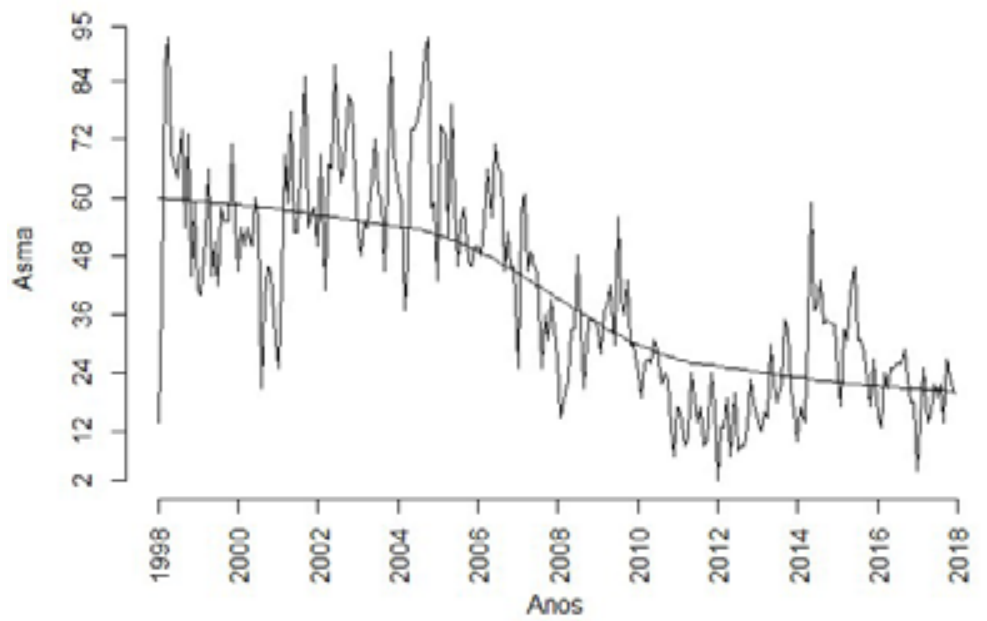

Fonte: Dados da pesquisa, 2019 oficial de domínio público, foi dispensado o encaminhamento ao Comitê de Ética e Pesquisa Científica (CEP). Portanto a atual pesquisa utilizou dados administrativos obtidos de documentação oficial de domínio público. Estes dados foram utilizados exclusivamente para objetivo desta pesquisa e as informações preparadas de maneira coletiva de tal forma que nenhum resultado se referiu a um indivíduo nominal, tampouco implicaram quais prejuízos para as pessoas ou instituições envolvidas.

Os dados meteorológicos foram organizados em planilhas do software Excel, juntamente com os registros de internações hospitalares por asma em crianças de 0 a 9 anos, e trabalhados pelo software estatístico R (R CORE TEAM, 2018), os quais foram tratados e analisados.

Em seguida, por se tratar de dados de contagem, após o ajuste do modelo de regressão de Poisson, foi necessário ajustar o modelo de regressão binomial negativa, mais adequado para tratar dados com variância superior à média condicional, foi elaborado com a adição de um novo parâmetro que reflete a heterogeneidade não observada.

\section{RESULTADOS}

Para avaliar a relação entre as variáveis meteorológicas e a ocorrência de asma, verificou-se, inicialmente o incremento de casos devido à sazonalidade. Os casos de asma no município de Campina Grande-PB, relativos aos dados do Sistema de Internação Hospitalar do Sistema Único de Saúde (SIH/SUS). Entre 1998 e 2017 foiregistrado um total de 9.701 internações, sendo 1.588 na faixa etária de 0 a 12 meses (16,3\%), 5.690 na faixa etária de $1 \mathrm{a}$ 4 anos $(58,6 \%)$ e 2.423 na faixa etária de 5 a 9 anos $(24,9 \%)$.

E em relação aos registros de asma, o maior número de internações ocorreu nos anos de 2002 e 2010, com um total de 807 e 810 registros respectivamente. A faixa etária que apresentou o maior número de internações foi de 1 a 4 anos em ambos os anos, já os meses de maiores registros foram maio e junho. E o ano de menor registro foi 2011 com 187 casos. 
Tabela 1. Estimativas dos parâmetros do modelo para ocorrência de internações hospitalares por asma no período de 1998 a 2017 em Campina Grande (PB).

\begin{tabular}{|c|c|c|c|c|c|}
\hline COEFICIENTES & ESTIMATIVA & ERRO & VALOR Z & P-VALOR & \\
\hline Intecepto ( $\beta 0)$ & 7,64360 & 1,35070 & 5,659 & $1,52 \mathrm{e}-08$ & $* * *$ \\
\hline Tmed ( $\beta 1)$ & 0,59868 & 0,11932 & 5,017 & $5,24 \mathrm{e}-07$ & $* * *$ \\
\hline $\operatorname{Tmax}(\beta 2)$ & $-0,31635$ & 0,05314 & $-5,953$ & 2,63e-09 & $* * *$ \\
\hline $\operatorname{Tmin}(\beta 3)$ & $-0,30808$ & 0,08943 & $-3,445$ & 0,000571 & $* * *$ \\
\hline UR ( $\beta 5)$ & $-0,03464$ & 0,00997 & $-3,474$ & 0,000513 & $* * *$ \\
\hline
\end{tabular}

Figura 2. Comportamento dos casos de asma em crianças menores de 9 anos em relação a temperatura média em Campina Grande (PB), 1998 a 2017.

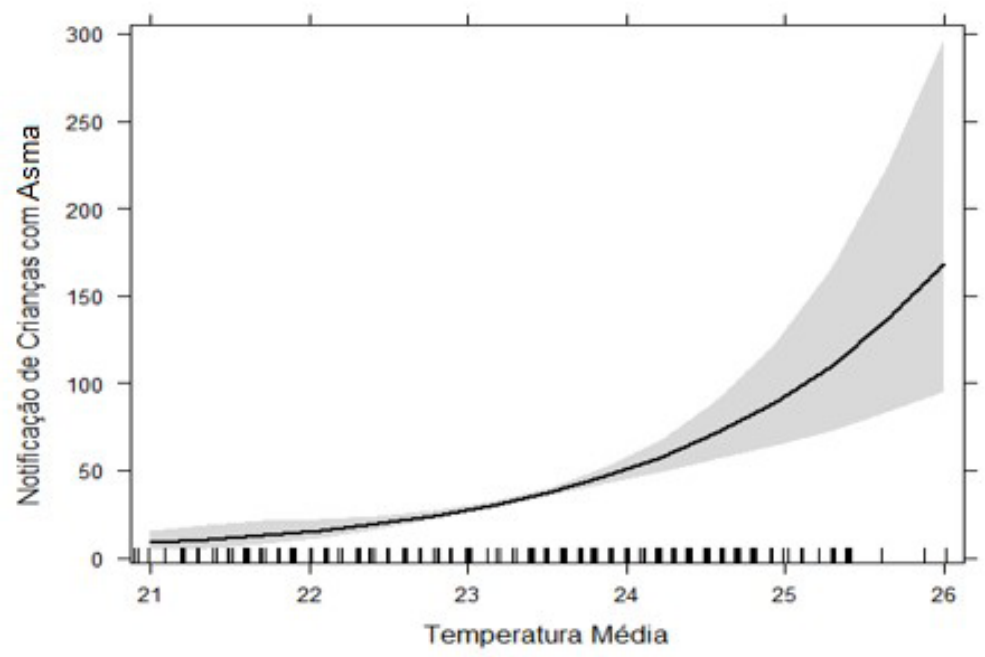

Fonte: Dados da pesquisa, 2019

Figura 3. Comportamento dos casos de asma em crianças menores de nove anos em relação à temperatura máxima em Campina Grande (PB), 1998 a 2017.

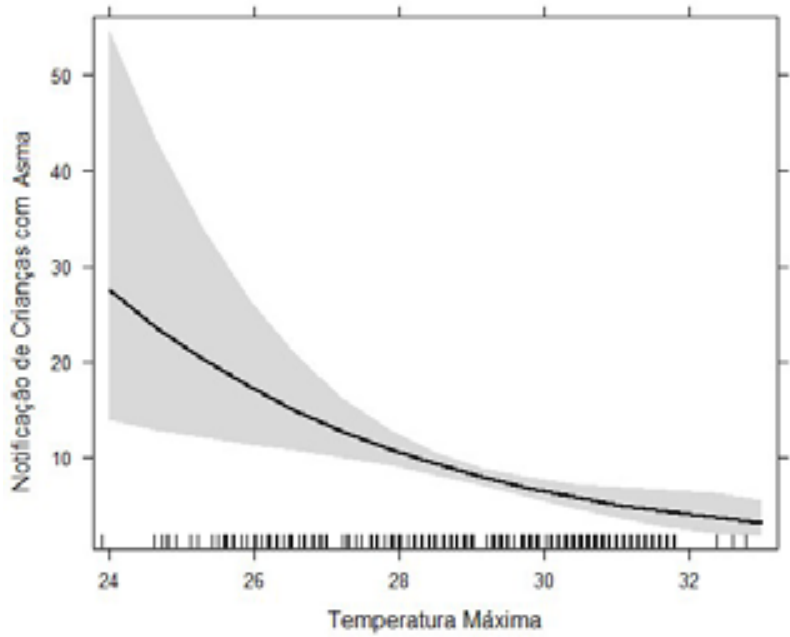

Fonte: Dados da pesquisa, 2019
Em 1998 ocorreram 737 casos de asma e em 2017, 219 casos, assim observa-se uma redução dos casos de asma durante o período de estudo (Figura 1). Percebeu-se, também, um efeito sazonal do número de casos registrados, com o maior número de registros entre abril a julho.

Em relação a análise estatística dos dados, como esses são de contagem utilizou-se, então, um modelo binomial negativo com ênfase na relação entre o número de hospitalizações de ocorrência de asma e as variáveis climáticas em estudo. Em que em que asma $\sim \mathrm{BN}(\mu \mathrm{i}, \phi)$ com ênfase na relação entre o número de hospitalizações de ocorrência de asmae as variáveis climáticas em estudo.

Os resultados apresentados na Tabela 1 indicam que as variáveis meteorológicas: temperatura média, máxima, temperatura mínima e umidade relativa, foram significativas ao nível de $5 \%$ de probabilidade, no que se referem à explicação da taxa de aumento/decréscimo nos casos de internações hospitalares por asma no período de 1998 a 2017 em Campina Grande (PB).

A atual pesquisa encontrou uma associação positiva entre os casos de asma e a temperatura média, já em relação à umidade relativa o atual estudo encontrou uma relação inversa, Figura 2.

$\mathrm{Na}$ atual pesquisa observou-se uma correlação inversa entre o número dos casos de asma e a variável temperatura máxima, Figura 3.

A atual pesquisa encontrou relação positiva coma temperatura média e inversa com a temperatura máxima e mínima. A variável precipitação não apresentou significância estatística.

Em relação a variável umidade relativa foi observado uma relação inversa em relaçãoao número de hospitalizações por asma, Figura 5.

\section{DISCUSSÃO}

A partir do estudo foi possívelobservar queparte da reduçãoem relação aos casos de asma ao longo dos anos, seja decorrente da consolidação do programa nacional de controle da asma. Em 2003 houve financiamento de medicamentos para asma 
Figura 4 - Comportamento dos casos de asma em crianças menores de nove anos em relação à temperatura mínima em Campina Grande (PB), 1998 a 2017.

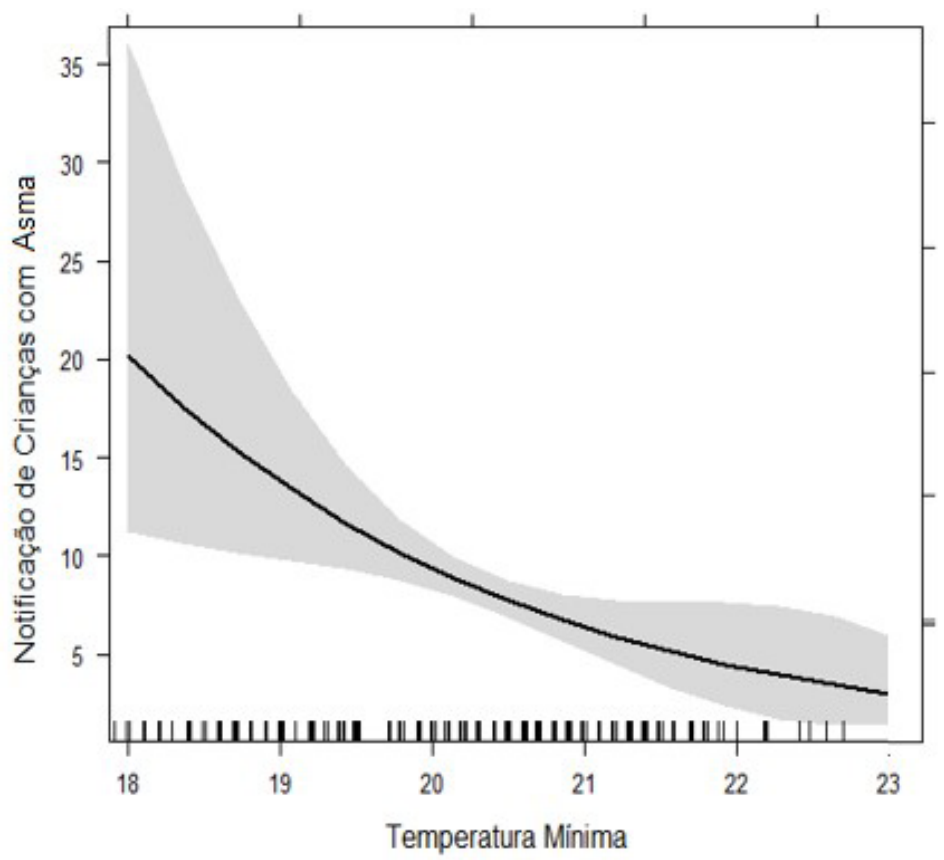

Fonte: Dados da pesquisa, 2019

Figura 5. Comportamento dos casos de asma em crianças menores de nove anos em relação à umidade relativa do ar em Campina Grande (PB), 1998 a 2017.

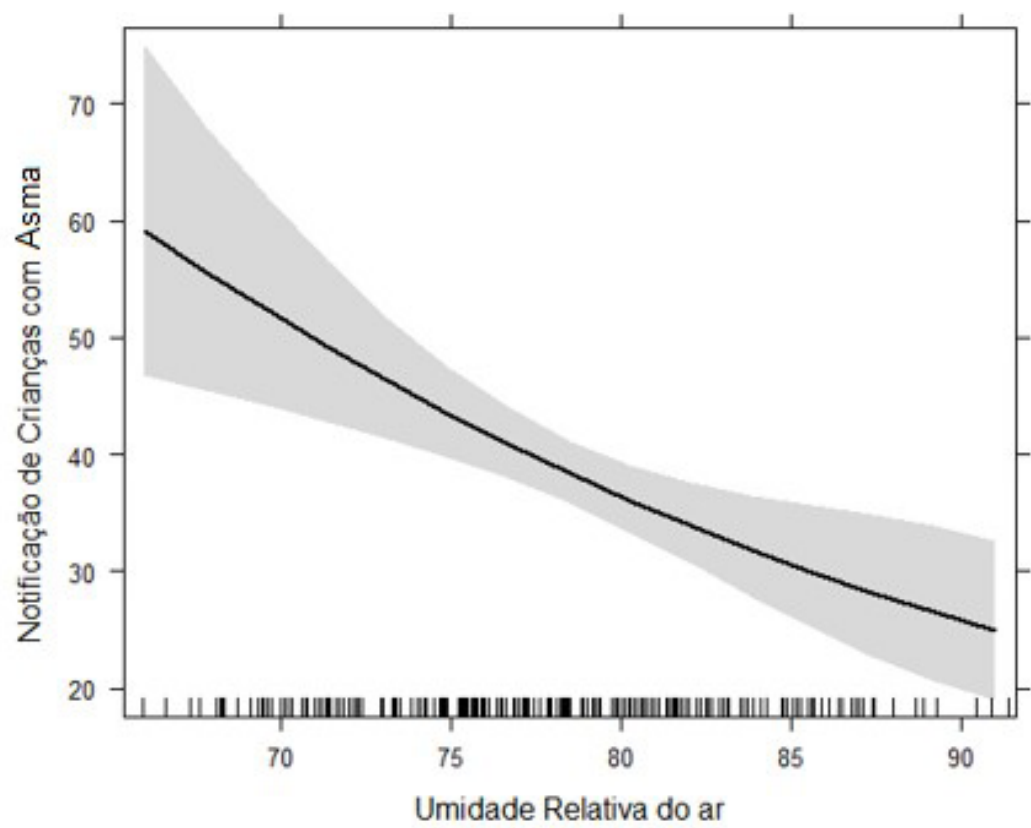

Fonte: Dados da pesquisa, 2019 grave, mas a partir de 2005 houve a aquisição de medicamentos para asma leve e moderada (portaria GM 2.084). Como a asma éuma doença inflamatória crônica de tratamento prolongado, no ano seguinte (2006), foi possível verificar o benefício da redução de exacerbações que poderiam levar a hospitalização ${ }^{(8,9)}$.

Quanto a taxa de aumento ou decréscimo nos casos de internações hospitalares por asma assim como nesta pesquisa. Valença, Restivo e Nunes ${ }^{(10)}$ observaram que os atendimentos, por asma abrangendo crianças em uma cidade satélite do Gama (DF), apresentavam uma variação sazonal, com um significativo aumento de casos em março e uma diminuição nos meses de agosto e setembro. Foi observado pelos pesquisadores que os casos agudos de asma aumentaram de um a dois meses, após a elevação da umidade relativa do ar e dois meses após o aumento do índice pluviométrico e da temperatura média.

Em relação aassociação positiva entre os casos de asma e a temperatura média e a relação inversa observada na umidade relativa observados neste estudo, corroboram com os resultados obtidos por González, Victora e Gonçalves ${ }^{(11)}$, que observaram em seus estudos em Pelotas, Rio Grande do Sul efeitos do clima (através da variável, temperatura média) no trimestre do nascimento e nos seis primeiros meses de vida sobre as internações por asma e pneumonia em crianças. Em relação às internações nos primeiros seis meses de vida constatou-se que a incidência de hospitalização por asma foi maior entre crianças que viveram os seis primeiros meses expostas a baixas temperaturas.

Estudos mostram a associação entre temperatura máxima e o número de casos de asma apresentou correlação positiva moderada e fraca nos anos de 2011 e $2012^{(12)}$. Entretanto correlações entre as variáveis ambientais e as morbidades em estudo não foram tão significativas.

De acordo com estudos realizados por Pesce ecolaboradores ${ }^{(13)}$ em cidades italianas. Observou-se na pesquisa que a prevalência nos casos de asma foi significativamente associada a variáveis climáticas (temperatura 
média anual, precipitação, radiação solar, ocorrendo um aumento nos casos de asma quando havia maior exposição a radiações solares e aumento da temperatura média anual e menores registros de precipitação).

A pesquisa de Silva e colaboradores ${ }^{(9)}$ avaliou o efeito da sazonalidade climática nas internações por asma. Os resultados mostraram uma relação inversa entre temperatura máxima e o número de hospitalizações por asma, bem como foi encontrado na atual pesquisa. Foi observada, também, uma relação positiva entre umidade relativa máxima e o número de hospitalizações por asma, diferentemente do atual estudo que encontrou uma relação inversa, Figura 5.

\section{CONCLUSÕES}

As variáveis meteorológicas: temperatura média, máxima, temperatura mínima e umidade relativa foram significativas, no que se refere à explicação dos casos de internações hospitalares por asma.Essas variáveis explicaram o aumento e decréscimo das internações hospitalares por asma.

\section{As variáveis}

meteorológicas:

temperatura

média, máxima,

temperatura mínima

e umidade relativa

foram significativas,

no que se refere

à explicação dos

casos de internações

hospitalares por

asma.
Pressupóe-se que ocorra um aumento de $81 \%$ nos casos de asma a cada grau de aumento da temperatura média, aumentando, do mesmo modo cerca de $27 \%$ desses casos com o aumento da temperatura máxima, já em relação a temperatura mínima espera-se que haja uma diminuição de $26 \%$ dos casos de asma em função do aumento da temperatura mínima. Estima-se, também, uma diminuição de $3 \%$ das internações por asma a medida que aumente a umidade relativa do ar.

Considerando a influência das variáveis meteorológicas (temperatura do ar e umidade relativa) nos registroshospitalares por asma. Torna-se possível avaliar o risco para a saúde da população em razão dos dados climáticos. Podendo ser planejadas ações que facilitem o atendimento e implementadas medidas preventivas de hospitalizações por asma na faixa etária em estudo evitando assim complicaçõesda referida morbidade, em períodos nos quais há uma intensidade de registros por asma que são no outono e inverno. -

\section{REFERÊNCIAS}

1. Hetzel JL, Silva LCC,Silva LMC. Asma Brônquica. I: Doenças Pulmonares. Guanabara Koogan: Rio de Janeiro: 2008.

2. Parker, S. Sistema circulatório. I: O livro do corpo humano. São Paulo: Ciranda Cultural, 2007.

3. Sociedade Paulista de Pneumologia e Tisiologia- SPPT. Pulmonar pela sua saúde respiratória [Internet]. 2012 [acesso em20jan2019]. Disponível em: http://www.pulmonar.org. $\mathrm{br} /$ ?op=paginas\&tipo=pagina\&secao=1\&pagina=35. 2012. Acesso: 20 jan. 2027.

4. Camelo NI,Solé D,Naspitz CK. Fatores de risco e evolução clínica da asma em crianças. Jornal de Pediatria. 1997; 73 (3): 15160.

5. Tian DD, Jiang $\mathrm{R}$, Chen XJ, Ye Q. Meteorologicalfactorsontheincidenceof MP and RSV pneumonia in children. PLoSOne.2017;12(3): e0173409.

6. Gern JE, Lemanske JRRF, BUSSE WW. Early life origins of asthma. The Journal of Clinical Investigation. 1999; 104 (7): 837-43.

7. Nunes BBdas, Mendes PC. Clima, ambiente e saúde: um resgate histórico. Caminhos de Geografia. 2012; 12(42): 258-269.

8. Cerci NA, Ferreira FOF, Bueno T. Exemplos brasileiros de programas de controle de asma. Jornal brasileiro de pneumologia. 2008; 34 (2): 103-106.
9. SilvaJLR, Diniz ITL, Nunes LFG, Borges TR. Efeito da sazonalidade climática nas hospitalizações por doença respiratória na cidade de Anápolis-GO entre 2002 e 2012. RevistaEducação Saúde. 2016; 4(2): 31-42.

10. ValençaLM, Restivo PCN, Nunes MS. Variação sazonal nos atendimentos de emergência por asma em Gama, Distrito Federal. Jornal Brasileiro de Pneumologia. 2006; 32 (4): 284-289.

11. González DA, Victora CG, Gonçalves H. Efeitos das condições climáticas no trimestre de nascimento sobre asma e pneumonia na infância e na vida adulta em uma coorte no Sul do Brasil. Caderno de Saúde Pública. 2008; 24(5): 1089-102, 2008.

12. Amorim JRG, Oliveira AM, NevesD,OliveiraGP. Associação entre variáveis ambientais e doenças respiratórias (asma e bronquite) em crianças na cidade Macapá-AP no período de 2008 a 2012. Planeta Amazônia: Revista Internacional de Direito Ambiental e Políticas Públicas. 2013; 5: 141-153.

13. PESCE, G.; BUGIANI, M.; MARCON, A.; MARCHETTI, P; CAROSSO, A.; ACCORDINI, S.; ANTONICELLI, L.; COGLIANI, E.; PIRINA, P.; POCETTA, G.; SPINELLI, F.; VILLANI, S.; MARCO, R. Geo-climatic heterogeneity in self- reported asthma, allergic rhinitis and chronic bronchitis in Italy. Science ofthe Total Environment. 2016; 544: 645-652. 